\title{
AUDITORIA DAS MALHAS DE CONTROLE DE UM FORNO DE TRATAMENTO TÉRMICO*
}

Pedro Henrique Moura Costa ${ }^{1}$

\section{Resumo}

Com o estudo realizado em artigos técnicos e acadêmicos e vivência no ramo industrial, ficou evidente uma deficiência no desempenho dos sistemas de controle regulatório das plantas industriais. Parte dessa deficiência é proveniente da falta de engenheiros de controle para auditar as malhas de controle regulatório. Foi realizado em um forno de tratamento térmico, um trabalho de auditoria das malhas de controle regulatório, baseando-se em uma metodologia já existente na literatura. Levantou-se informações do processo do forno como, os controladores automáticos, o sistema de aquisição de dados e a infraestrutura de rede de automação e por seguinte, as implementações dos PID's no CLP. Após essa contextualização, métricas para avaliar o desempenho inicial das malhas foram utilizadas e então, o funcionamento dos elementos de controle utilizados no forno foram diagnosticados através de ferramental estatístico e análise dos sinais na ferramenta PIMS. Adquirido o conhecimento necessário sobre o processo e o diagnosticado de maneira concisa, ações corretivas e de otimização foram realizadas e propostas com o intuito de otimizar o desempenho das malhas de controle. Por fim, os índices de desempenho do sistema são recalculados e os valores comparados com os valores pré auditoria, concluindo que pode ser possível melhorar alguns dos principais termos adotados no negócio como redução de custos, aumento de produtividade e sustentabilidade.

Palavras-chave: Controlador PID; Auditoria de malhas; Otimização de processo.

\section{AUDIT OF CONTROL LOOPS OF A STEEL HEAT TREATMENT FURNACE Abstract}

With the study on technical and academic and experience in the industrial field, articles became apparent deficiency in the performance of regulatory control systems of industrial plants. Part of this deficiency comes from the lack of control engineers to audit the regulatory control loops. Was performed in a heat treatment furnace, an audit of regulatory control loops, based on a methodology in existing literature. Rose information furnace process as automatic controllers, data acquisition system and the entire network infrastructure the, automation and following, the implementations of PID in the PLC. After this contextualization, metrics to evaluate the performance of the initial meshes were used and then, the operation of the controls used in the oven were diagnosed by statistical tools and analysis of signals in PIMS. Acquired the necessary knowledge about the process and concise diagnosed, corrective actions and optimization way proposed in order to optimize the operation of the furnace control loops were performed. Finally, the index that quantify system performance values are recalculated and compared with the pre audit figures, concluding that it may be possible to improve some of the key terms adopted in the business as reduced costs ,increased productivity and sustainability.

Keywords: PID controller; Audit of control loop; Process optimization.

1 Engenheiro de Controle e Automação, Bacharel, Analista de Sistemas, TI/PIMS, TSA - Tecnologia de Sistemas de Automação, Belo Horizonte, Minas Gerais, Brasil.

\footnotetext{
* Contribuição técnica ao $18^{\circ}$ Seminário de Automação e TI Industrial, 23 a 26 de setembro de 2014, São Paulo, SP, Brasil.
} 


\section{INTRODUÇÃO}

Com a alta competitividade existente no mercado, é extremamente importante melhorar alguns aspectos de produção, afim de garantir a competitividade para com as demais empresas do mesmo ramo. Com essas métricas à serem alcançadas pelo setor industrial, a necessidade da manutenção de um bom controle automático do processo através da auditoria das malhas de controle está cada vez mais consolidada. Um sistema de controle confiável, permite que o processo opere próximo aos limites ótimos de operação, garantindo segurança e respeito às normas ambientais vigentes, consequentemente o mesmo estará em uma condição operacional favorável o que maximiza os lucros na produção [1].

Apesar dos benefícios, sabe-se que hoje na implantação de novos sistemas de controle regulatório nem todas as etapas necessárias para implantação são realizadas, devidos a tempo e custos reduzidos, além de incertezas sobre o comportamento final do processo, dificultando à adoção de um melhor ponto operacional [2]. Essas dificuldades na implantação e também da falta de manutenção dos elementos constituintes de uma malha de controle automático, torna-as ineficiente e não são alcançados desempenhos ótimos das mesmas. Dessa forma, surge a necessidade de realizar-se constantemente a avaliação do desempenho das malhas de controle, trabalho realizado pelo Engenheiro de Controle de Processos através de auditoria das malhas de controle.

Segundo [1] e [3], auditoria de malhas de controle, se traduz em fazer uma análise constante das propriedades específicas do controlador que reflita no desvio do seu desempenho final, avaliar a saúde da válvula de controle, diagnosticar e apontar as causas das perturbações na planta como um todo, além de estudar o comportamento de todos os elementos envolvidos no controle (sensores, atuadores e controladores) com as interações existentes entre os controladores. Com esse conhecimento, é necessário levantar as ações que podem interferir no funcionamento das malhas de controle.

Esse trabalho de auditoria necessita ser periódico, contínuo e realizado por profissionais capacitados para tais atividades [2]. Infelizmente, esse trabalho ainda não é uma rotina na maioria das empresas no ramo industrial e os principais motivos para falta da realização desses trabalhos são [4]:

Falta de engenheiros nas companhias para realização da auditoria/otimização dos ativos e falta de treinamento dos engenheiros e operadores para agir proativamente diante da perda de performance dos ativos.

Como consequência da falta dessa atividade nas indústrias, tem-se no cenário atual o desempenho dos sistemas como mostra a pesquisa realizada e inserida abaixo [2]:

Tabela 1 - Dados referentes ao cenário dos controladores PID's na indústria atual

\begin{tabular}{lc}
\hline \multicolumn{1}{c}{ Problemas típicos das malhas de controle } & $\begin{array}{c}\text { Percentual das malhas } \\
\text { auditadas }\end{array}$ \\
\hline Válvulas de controle com problemas & $30 \%$ \\
Problemas de sintonia (parâmetros incoerentes) & $30 \%$ \\
Problemas de sintonia (comprometimento do desempenho) & $85 \%$ \\
Estratégia de controle inadequada & $15 \%$ \\
Controlador em modo manual & $30 \%$ \\
Desempenho insatisfatório da malha & $85 \%$ \\
Malhas com melhor desempenho em automático que manual & $25 \%$ \\
\hline
\end{tabular}

A partir dos dados exibidos acima, apenas $25 \%$ das malhas que operam em modo automático possuem desempenho melhor do que em modo manual, $85 \%$ dos controladores possuem sintonia inadequada e $85 \%$ das malhas estão com

\footnotetext{
* Contribuição técnica ao $18^{\circ}$ Seminário de Automação e TI Industrial, 23 a 26 de setembro de 2014, São Paulo, SP, Brasil.
} 
desempenho insatisfatório. Ou seja, é notório a real necessidade da constante avaliação de desempenho das malhas de controle de um processo industrial.

Pelos cenários apresentados acima, realizou-se um trabalho de auditoria nas malhas de controle existentes num forno de tratamento térmico de uma usina Siderúrgica.

O principal objetivo é melhorar a regulação e servomecanismo dos controladores automáticos do processo do forno de tratamento térmico, com a hipótese de melhorar as avaliações realizadas em laboratório através da melhoria na qualidade do produto final.

\section{MATERIAIS E MÉTODOS}

\subsection{Sintonia de Controlador PID}

O método que foi adotado para sintonia dos controladores PID do processo estudado nesse trabalho, é o Síntese Direta para controladores PI e PID.

A escolha desse método foi devido à relativa simplicidade de implementação e principalmente pela possibilidade de estabelecimento da dinâmica da resposta do sistema em malha fechada.

A síntese direta estabelece uma relação direta entre controlador e processo a ser controlado [5].

Seu princípio é o cancelamento do(s) pólo(s) do processo, erro nulo em estado estacionário com a alocação de um pólo e garantia de uma resposta em malha fechada desejada.

$$
C(s)=\frac{1}{\lambda s}\left(\frac{1}{G(s)}\right)
$$

A equação 1, tem-se a Função de Transferência do controlador.

$\mathrm{Gc}(\mathrm{s})$ é a função de transferência do controlador, $\mathrm{G}(\mathrm{s})$ a função de transferência do processo.

A tabela 2 mostra a relação entre as funções de transferência dos processos supracitados com os do respectivo controlador.

Tabela 2 - Parâmetro de sintonia de controladores PI e PID pelo método da síntese direta

\begin{tabular}{|c|c|c|c|}
\hline & $G(s)$ & Parametros de Sint & tonia \\
\hline 1 ordem & $G(s)=\frac{K e^{-\theta s}}{(\tau s+1)}$ & $K c=\frac{\tau}{K \lambda}$ & $\Gamma_{i}=\tau$ \\
\hline $\begin{array}{l}2^{a} \text { ordem } \\
\text { sobreamortecido }\end{array}$ & $G(s)=\frac{K e^{-\theta s}}{\left(\tau_{1} s+1\right)\left(\tau_{2} s+1\right)}$ & $K c=\frac{\left(\tau_{1}+\tau_{2}\right)}{K \lambda}$ & $T_{d}=\frac{\tau_{1} \tau_{2}}{\left(\tau_{1}+\tau_{2}\right)}$ \\
\hline $\begin{array}{l}2^{*} \text { ordem } \\
\text { subamortecido }\end{array}$ & $G(s)=\frac{K \omega_{n}^{2} e^{-\theta s}}{s^{2}+2 \zeta \omega_{n} s+\omega_{n}^{2}}$ & $K c=\frac{2 \zeta}{\lambda K \omega_{n}}$ & $T_{d}=\frac{1}{2 \zeta \omega_{n}}$ \\
\hline
\end{tabular}

\subsection{Objetivos de Controle}

No desenvolvimento do projeto dos controladores, é muito importante entender o objetivo do sistema que será controlado dentro do processo, pois, é fundamental que o controlador atenda todas as necessidades desse sistema e especificações do processo. Conforme [6] segue abaixo alguns principais objetivos de controle:

Segurança: a malha sempre está com o sinal da variável controlada CO saturada, visto que todo processo possui um limite mínimo ou máximo aceitável e a PV deve ficar sempre dentro dessa faixa, com isso, o controlador atuará apenas quando a PV violar algum dos limites estabelecidos.

* Contribuição técnica ao $18^{\circ}$ Seminário de Automação e TI Industrial, 23 a 26 de setembro de 2014, São Paulo, SP, Brasil. 
Servomecanismo: são malhas que o sinal de referência SP é variável. A função dessas malhas é rastrear o SP variável.

Regulação: são malhas que o SP é constante e objetivam manter a variável controlada estável sobre o valor do SP. É necessário que sejam eficientes também na eliminação de perturbações de carga e distúrbios.

\subsection{Métodos para Realização de Auditoria de Malhas de Controle}

O trabalho de auditoria torna o auditor um conhecedor do processo auditado com capacidade de definir estratégias e ações a serem tomadas sobre os elementos de uma malha de controle (controlador, sensor e atuador), vislumbrando a melhoria dos objetivos do controle automático.

Para este trabalho, foi adotada uma metodologia para auditoria de malhas de controle de processos que foi proposta e utilizada por [3], e é composta por quatro etapas distintas conforme descritas abaixo:

\subsubsection{Fase I - levantamento de campo}

Na primeira fase, é necessário analisar o processo em si, o sistema de controle e a operação da planta. Para realização dessa etapa, alguns documentos podem ser coletados e algumas atividades podem ser realizadas pelo auditor para ambientação com a unidade produtora. Estão relacionados abaixo algumas ferramentas que possibilitam essa ambientação com a unidade de produção:

Estudo da documentamação de engenharia existente, imagens do processo através de visitas e fotografias, estudo do sistema de controle e automação do processo, ambientação com o processo e rotina de operação e levantamento sobre ferramentas computacionais para monitoramento de malhas, historiadores de processo (PIMS).

Com essas informações levantadas durante o processo de levantamento de campo, são sugeridos por [3] alguns documentos que podem ser elaborados e servirão de auxílio na realização das próximas auditorias: memorial descritivo do processo, lista das malhas de controle, descritivo das malhas, identificação das principais condições de operação da planta e levantamento de indicadores econômicos.

\subsubsection{Fase lla - avaliação inicial de desempenho}

Para a realização de qualquer trabalho de otimização de controladores de processo PID, é necessário quantificar o desempenho das malhas de controle. Para tal, foram desenvolvidas métricas que as quantificam de forma determinística. Mas, de acordo com [8] essa análise é diferenciada por diferentes pontos de vista onde, por exemplo, para um Engenheiro de Controle uma malha em boas condições é uma malha que consiga rejeitar de forma rápida as perturbações, que siga bem uma referência e seja preciso, diferentemente para o gerente onde, retornos econômicos através de redução de consumo de insumos energéticos, redução de custos de manutenção traduzem em um bom desempenho.

Não existe uma métrica padrão para determinação de quais índices devem ser utilizados para os respectivos controladores. Foi proposto por [3] que se escolha os índices baseados nos respectivos objetivos de controle das malhas. Segue abaixo a tabela 3, os relacionando:

Tabela 3 - (modificado) aplicação dos índices por objetivo.

\footnotetext{
* Contribuição técnica ao $18^{\circ}$ Seminário de Automação e TI Industrial, 23 a 26 de setembro de 2014, São Paulo, SP, Brasil.
} 


\section{AUTOMAÇÃO \& TI}

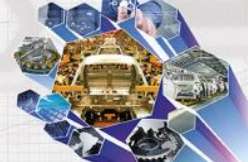

\begin{tabular}{cccc}
\hline & $\begin{array}{c}\text { Controle } \\
\text { Regulatório }\end{array}$ & $\begin{array}{c}\text { Controle Servo } \\
\text { Mecanismo }\end{array}$ & Malha Segurança \\
\hline varPV & $\mathrm{X}$ & & \\
IEM & $\mathrm{X}$ & $\mathrm{X}$ & $\mathrm{X}$ \\
ExcCO & $\mathrm{X}$ & & \\
Limite violado & $\mathrm{X}$ & & \\
\hline
\end{tabular}

Variabilidade relativa da PV (varPV): mede a dispersão da variável controlada em desvio padrão de um conjunto de dados amostrados.

$$
\% \operatorname{varPV}=3 *\left(\sqrt{\frac{\sum_{i=1}^{n}(x i-\bar{x})^{2}}{n-1}}\right)
$$

Integral do erro médio - IEM: Somatório do sinal do erro de controle acumulado ao longo de um período.

$$
\mathrm{IEM}=\frac{\sum_{i=1}^{n}(S P i-P V i)}{n}
$$

Excursão da CO: Somatório de todos os incrementos do sinal que chega ao atuador num determinado intervalo. Desconsidera as incrementações devido aos ruídos pertencentes ao sinal.

Limite violado: Índice que indica a violação da variável controlada sobre os limites estabelecidos para o processo.

Índices para resposta ao degrau:

Percentual de overshoot ou sobressinal: é o percentual de que o valor máximo do sinal da resposta do sistema ultrapassa o valor em estado estacionário.

$$
\mathrm{PO}=100 *\left(\frac{\mathrm{y}(\mathrm{tp})-\mathrm{y}(\infty)}{\mathrm{y}(\infty)}\right)
$$

Tempo de acomodação: Mede o tempo de acomodação do sistema.

Oscilação: Diz-se segundo [3] que uma malha é dita como oscilatória quando a tendência da variável controlada apresenta um comportamento periódico com um padrão regular de desvios em torno da variável de referência.

\subsubsection{Fase II - diagnósticos preliminares}

É de suma importância um estudo completo sobre as interações existentes no equipamento, perturbações e também da saúde dos atuadores existentes no processo. Esse esforço possibilita um direcionamento dos pontos onde o engenheiro de Controle necessitará focar mais os trabalhos para melhorar o desempenho da planta.

Foram adotadas nesse trabalho a matriz de correlação que segundo [8] mede o grau de intercorrelação entre sinais e foi também utilizada a ferramenta historiadora de dados de processo Plant Information Management System (PIMS). O PIMS torna-se um facilitador para estudo do comportamento do processo em momentos distintos e com isso, facilita na identificação de falhas e visualização de melhorias à serem aplicadas, servindo também como principal fonte de dados para utilização nos algoritmos computacionais que por exemplo gerarão a matriz de correlação[13].

$\mathrm{Na}$ matriz de correlação normalizada de -1 a 1 , valores maior do que 0,7 indicam um alto acoplamento direto entre as variáveis. Para valores menores do que $-0,7$ temos um alto acoplamento inverso entre as variáveis. Os resultados são exibidos apenas abaixo da diagonal principal, pois, a matriz de correlação é simétrica [3].

* Contribuição técnica ao $18^{\circ}$ Seminário de Automação e TI Industrial, 23 a 26 de setembro de 2014 , São Paulo, SP, Brasil. 


\subsubsection{Fase III - otimização e ações corretivas}

O termo otimização nesse trabalho é designado à redução da variabilidade do processo com a adoção de estratégias de controle que reduzam ou eliminem as perturbações que afetam a variável controlada.

Algumas ações que visam à otimização do sistema de controle e consequentemente da produção estão listadas abaixo segundo [3]:

Revisão com a aplicação de estratégias de controle (controle seletivo, controle em razão, compensação direta, faixa dividida, controle em cascata dentre outras), substituição e manutenção de sensores e atuadores, sintonia de controladores e revisão da implementação dos controladores no CLP ou SDCD.

Como fase da otimização, para determinação das equações matemáticas que se aproximam da dinâmica real do processo, passo necessário para sintonia dos controladores, foi utilizado o método da caixa preta que é bastante atrativo devido à dificuldade na obtenção de um tratamento matemático que descreva o processo dinamicamente através de equações diferenciais [9].

Mas esse teste necessita ser criterioso e de acordo com [10], as seguintes ações devem ser realizadas para os testes que servirão para modelar matematicamente o processo e consequentemente utilizar do modelo para otimização do mesmo:

Energizar a instrumentação da planta, ajustar as referências para os valores de operação do processo, esperar a estabilização das malhas antes da realização do teste, alterar as malhas de controle para o modo manual, ajustando a variável controlada em um valor dentro da faixa de operação, aplicar degrau ou pulso à variável manipulada e registrar as alterações nas variáveis controladas de cada malha.

\subsubsection{Reprojeto dos parâmetros de sintonia do controlador}

De posse da função matemática que representa a dinâmica do processo, para definir os novos parâmetros de sintonia deve-se primeiro escolher um método de sintonia que atenda aos objetivos desejados para a resposta do sistema em malha fechada [3].

Após definido o método, deve-se ter estabelecido alguns critérios de desempenho do sistema em malha fechada como exemplo, tempo de acomodação, possibilidade de ter ou não sobressinal na variável controlada e etc.

De posse dessas informações, é necessário verificar qual algoritmo do controlador é utilizado no CLP (paralelo, série, ideal) para então calcular os parâmetros do controlador.

\section{RESULTADOS E DISCUSSÃO}

\subsection{Fase I: Levantamento de Campo}

\subsubsection{Descrição do processo}

No processo de tratamento térmico, ocorre um conjunto de operações de Aquecimento do aço onde algumas variáveis devem ser rigorosamente controladas. Nesse forno, o tratamento possui duas etapas: Aquecimento e Patamar. Na fase de Aquecimento, existe uma rampa de temperatura que deve ser seguida afim de que não sejam provocados defeitos no material e na fase de patamar, deve-se manter a tempera em um valor constante para a finalização do tratamento.

O objetivo desse tratamento é a obtenção de uma microestrutura que proporcione propriedades elevadas de dureza e resistência mecânica ao aço tratado.

\footnotetext{
* Contribuição técnica ao $18^{\circ}$ Seminário de Automação e TI Industrial, 23 a 26 de setembro de 2014, São Paulo, SP, Brasil.
} 
A análise dessa microestrutura do material é posteriormente realizada em laboratório, via microscópio.

\subsubsection{Malhas de controle}

Nesse forno, é necessário o controle de seu ambiente interno para garantia da qualidade das peças metálicas produzidas, para isso, existem alguns sinais que são parte desse controle: temperatura, pressão atmosférica e concentração de oxigênio. Abaixo segue uma tabela com informações detalhadas de cada malha utilizada no processo para seu controle.

Tabela 4: Tabela de informações detalhadas das malhas de controle

\begin{tabular}{|c|c|c|c|c|c|c|}
\hline & PIC 223A & TIC 410A & TIC 411A & TIC 410A & TIC 411A & AIC 225A \\
\hline Estratégia & Feedback & Feedback & Feedback & Feedback & Feedback & Feedback \\
\hline Objetivo & Segurança & Servomecanismo & Servomecanismo & Regulação & Regulação & Regulação \\
\hline Atuador & FCV223A & $\mathrm{AQ410A}$ & $\mathrm{AQ411A}$ & AQ410A & $\mathrm{AQ411A}$ & FCV225A \\
\hline Sensor & РT223A & TT226A & ТT223A & TT410A & TT411A & UT226A \\
\hline Faixa de medição & & o.a $1200^{\circ} \mathrm{C}$ & 0 a $1200^{\circ} \mathrm{C}$ & 0 a $1200^{\circ} \mathrm{C}$ & 0 a $1200^{\circ} \mathrm{C}$ & O. A $100 \%$ \\
\hline Faixa de operação & & 900 a $1000^{\circ} \mathrm{C}$ & 900 a $1000^{\circ} \mathrm{C}$ & 900 a $1000^{\circ} \mathrm{C}$ & 900 a $1000^{\circ} \mathrm{C}$ & 60 a $90 \%$ \\
\hline Faixa de alarme & $>25 \mathrm{mmCA}$ & $>1100^{\circ} \mathrm{C}$ & $>1100^{\circ} \mathrm{C}$ & $>1100^{\circ} \mathrm{C}$ & & 60 a $90 \%$ \\
\hline
\end{tabular}

\subsubsection{CLP}

Identificou-se o CLP utilizado para o controle automático do processo, o algoritmo dos blocos PID's, a implementação desses blocos nas respectivas rotinas de execução e a lógica implementada.

\subsection{Fase lla: Avaliação de Desempenho}

Devido à maior prioridade da malha AIC225A, onde o potencial de retorno em qualidade do produto é grande, os índices de desempenho pré e pós otimização foram focados à priori para a respectiva malha, apesar do trabalho ter sido realizado em todo o processo produtivo do forno.

Foram coletadas informações da AIC225A através do sistema Planttriage como base de dados dos cálculos dos índices de desempenho da malha descritos na tabela 3, de acordo com o respectivo objetivo de controle.

Variabilidade relativa da PV (varPV): $2,49 \%$

Integral do erro médio - IEM: -0.065582\%

Excursão da CO: 2.8909 aberturas/dia

Índices para resposta ao degrau:

Tempo de acomodação: 51 minutos

\subsection{Fase II: Diagnósticos Preliminares}

Na fase IIA, foram utilizados para diagnosticar o funcionamento do forno a matriz de correlação calculada e inserida abaixo e a análise temporal das variáveis do forno através do PIMS.

\footnotetext{
* Contribuição técnica ao $18^{\circ}$ Seminário de Automação e TI Industrial, 23 a 26 de setembro de 2014 ,
} São Paulo, SP, Brasil. 


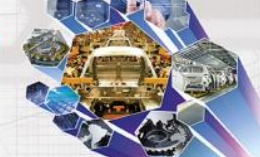

Tabela 5: Matriz de correlação.

\begin{tabular}{ccccccc}
\hline & CO TIC 410 & PV TIC 410 & CO AIC 225A & PV 225A & Temp. vapor PV PIC 223A \\
\hline CO TIC 410 & 1 & $-0,7178$ & 0,9428 & $-0,8999$ & 0,3305 & 0,4712 \\
PV TIC 410 & $-0,7178$ & 1 & $-0,5107$ & 0,4649 & $-0,4599$ & $-0,3063$ \\
CO AIC 225A & 0,9428 & $-0,5107$ & 1 & $-0,9854$ & 0,203 & 0,7663 \\
PV 225A & $-0,8999$ & 0,4649 & $-0,9854$ & 1 & $-0,1316$ & $-0,6551$ \\
Temp. vapor & 0,3305 & $-0,4599$ & 0,203 & $-0,1316$ & 1 & 0,0761 \\
PV PIC 223A & 0,4712 & $-0,3063$ & 0,7663 & $-0,6551$ & 0,0761 & 1 \\
\hline
\end{tabular}

A análise do resultado final da matriz de correlação, pode ser traduzida da seguinte forma: valores entre 0,7 à 1 indicam forte acoplamento positivo entre os sinais. Valores entre -1 à $-0,7$ indicam forte acoplamento negativo entre os sinais.

Não foi possível realizar testes para quantificação de agarramento, histerese e curva de resposta dos atuadores FCV223A e FCV225A devido à falta de instrumentos de medição de vazão nas linhas.

\section{TIC 410 e TIC 411}

No período de Aquecimento, identificou-se a existência de acoplamento mútuo entre os aquecedores à resistência elétrica linear de potência variável AQ411A e AQ410A que se localizam na parte inferior e superior do forno respectivamente do forno.

$\mathrm{Na}$ fase de Patamar, além do primeiro acoplamento existente no Aquecimento, têmse dois controladores atuando em uma mesma PV (TIC410A e TIC411A).

Esse cenário pode ser perigoso, pois, um controlador pode entrar em conflito com a ação executada pelo outro, tornando a malha oscilatória em alguma condição de operação. Como perturbação, tem- se o vapor que é adicionado no interior do forno em uma temperatura bem abaixo do ambiente interno.

AIC 225A

De acordo com a matriz de correlação e estudo do processo via PIMS, identificou-se a existência de três acoplamentos que devem ser considerados no projeto do controlador: o sinal da CO do PIC223A e principalmente o sinal da PV das TIC's 410A e 411 A.

Como pode ser visto também no gráfico retirado do Planttriage, identificou-se um comportamento de duas dinâmicas atuando sobre o sinal da PV. Essa situação pode ser vista no gráfico abaixo:

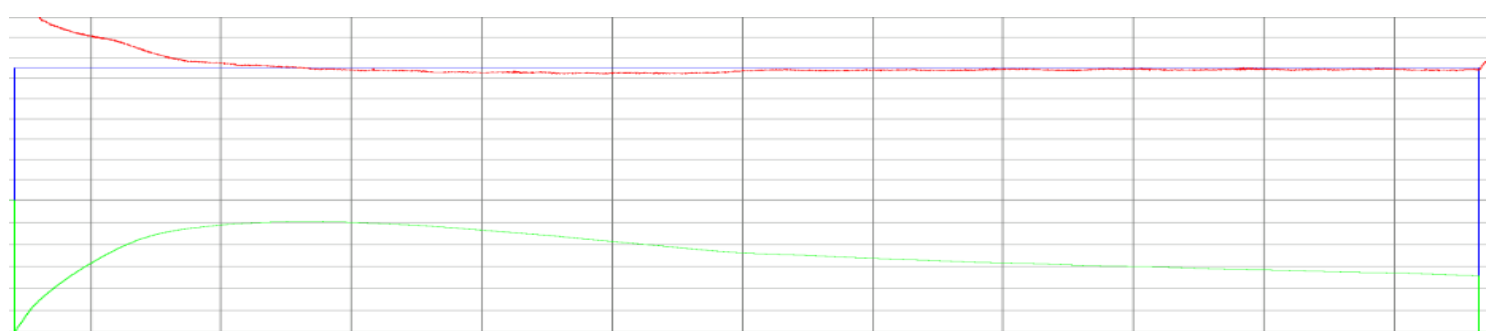

Figura 1 - Comportamento dinâmico da AIC225A (pena vermelha - PV, pena verde - CO, pena azul $-\mathrm{SP})$

A pena vermelha trata-se da PV, a pena verde do sinal da CO e a azul o SP.

Para esse comportamento foram levantadas duas hipóteses, mas baseado nos estudos do processo e na constituição física do forno, foi sustentada a hipótese abaixo:

Dado a estrutura física do forno que possui vedação da parte inferior à base de areia e orifício que mantém contanto com o ambiente externo a excursão da válvula pode

* Contribuição técnica ao $18^{\circ}$ Seminário de Automação e TI Industrial, 23 a 26 de setembro de 2014, São Paulo, SP, Brasil. 
ser para compensação de uma perturbação constante sobre a PV com característica integradora.

Com a realização de um teste em malha aberta no sistema, observou-se a presença de uma banda morta na válvula na faixa compreendida de $0 \%$ a $10 \%$, pois, só há passagem de vapor quando sua abertura ultrapassa esse limite máximo.

Apesar de todas as especificidades da malha, a principal dificuldade de controle é a não linearidade do processo, que, possui pelo menos dois ganhos que ultrapassam de dez vezes a relação entre eles.

Essa não linearidade é devido ao processo de concentração de oxigênio onde, a agregação de $\mathrm{O} 2$ ao meio possui dois momentos bem distintos pré e pós saturação desse meio medido pelo coeficiente de solubilidade.

Mesmo assim, no cenário atual o sistema está sendo controlado utilizando o controle clássico que o considera como linear. O maior problema é que essa consideração pode afetar o desempenho do controlador dependendo do ponto de operação da planta [11] e esse problema é visualizado em alguns tratamentos, onde, em certo momento percebe-se um comportamento oscilatório da malha de controle.

\subsection{Fase III: Otimização e Ações Corretivas}

Depois de realizadas as etapas I, II e Ila, foram divididas as ações de correção e otimização em dois grupos: ações realizadas e ações propostas.

A necessidade dessa divisão foi devido à falta de tempo necessário para realização de todas as atividades até o momento.

Das ações realizadas, foram feitas as seguintes atividades:

1a - Revisão da implementação do controlador AIC 225A na lógica de funcionamento do forno no CLP.

Periodicidade de execução do cálculo PID: deixou-se como comentário para equipe de automação do processo que o bloco PID está em uma rotina cíclica de execução de 100ms no CLP, porém, a periodicidade do cálculo está configurada para 1s. Esse parâmetro tem que ser fixo e preferencialmente igual ao período de execução do bloco para que não haja variações no resultado final dos parâmetros Ti e Td de acordo com o esperado [12].

$2^{\text {a }}$ - Revisão da aplicação da estratégia de controle aplicado à todo o tratamento.

Para redução do tempo de acomodação da malha no início do tratamento, em um primeiro momento foi adotado um controlador com parâmetros intermediários, analisado os ganhos obtidos e as possibilidades de melhora e como trabalho futuro, será projetado um controlador chaveado que possui resultados favoráveis à utilização deste ao invés de um controlador intermediário [11].

$3^{\text {a }}$ - Sintonia de controladores: otimização dos parâmetros de sintonia para melhorar o desempenho.

Foram realizados testes em malha aberta no processo para determinação do modelo matemático que se aproxime ao máximo do real para descrever a dinâmica do processo.

Devido à não linearidade do processo, os testes para modelamento ocorreram no início e quando o processo já se encontrava próximo do valor desejado.

Das hipóteses levantadas sobre as dinâmicas em paralelo existentes, a adotada devido à construção física do forno, análises de testes que foram realizados e simulações desenvolvidas no Matlab, a melhor representação em diagrama de blocos ficou conforme abaixo:

\footnotetext{
* Contribuição técnica ao $18^{\circ}$ Seminário de Automação e TI Industrial, 23 a 26 de setembro de 2014 ,
} São Paulo, SP, Brasil. 


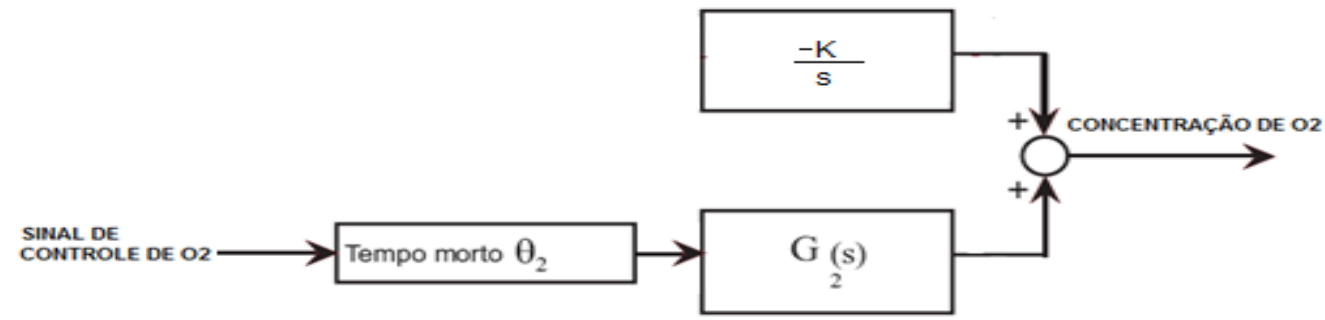

Figura 2 - Diagrama de blocos representativo da soma de um sistema $\mathrm{G}(\mathrm{s})$ com um sistema integrador não controlado

Após modelagem do processo nas duas etapas, abaixo estão os parâmetros de primeira ordem encontrados e as curvas com o modelo real x calculado.

Tabela 6: Parâmetros do processo controlado pelo AIC 225A.

\begin{tabular}{cccc}
\hline & $\mathbf{K p}$ & $\boldsymbol{\tau}$ & $\boldsymbol{\Theta}$ \\
$\begin{array}{c}1^{\mathrm{a}} \text { parte }- \text { Início } \\
\text { tratamento }\end{array}$ & $-0,68$ & $25,38 \mathrm{~s}$ & $6,32 \mathrm{~s}$ \\
$2^{\mathrm{a}}$ parte & $-0,052$ & $36,47 \mathrm{~s}$ & $6,47 \mathrm{~s}$ \\
\hline
\end{tabular}

\section{1- parte}

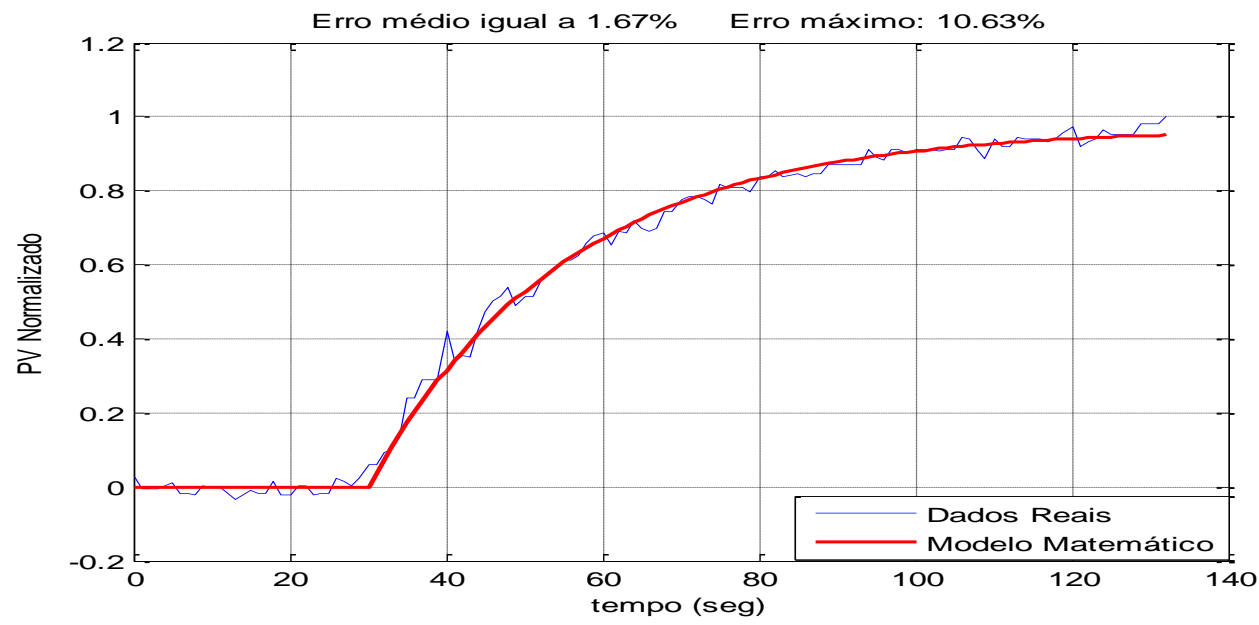

Figura 3 - Modelo real x matemático da parte I obtido para modelagem do controlador.

\section{$2^{\mathrm{a}}$ parte}

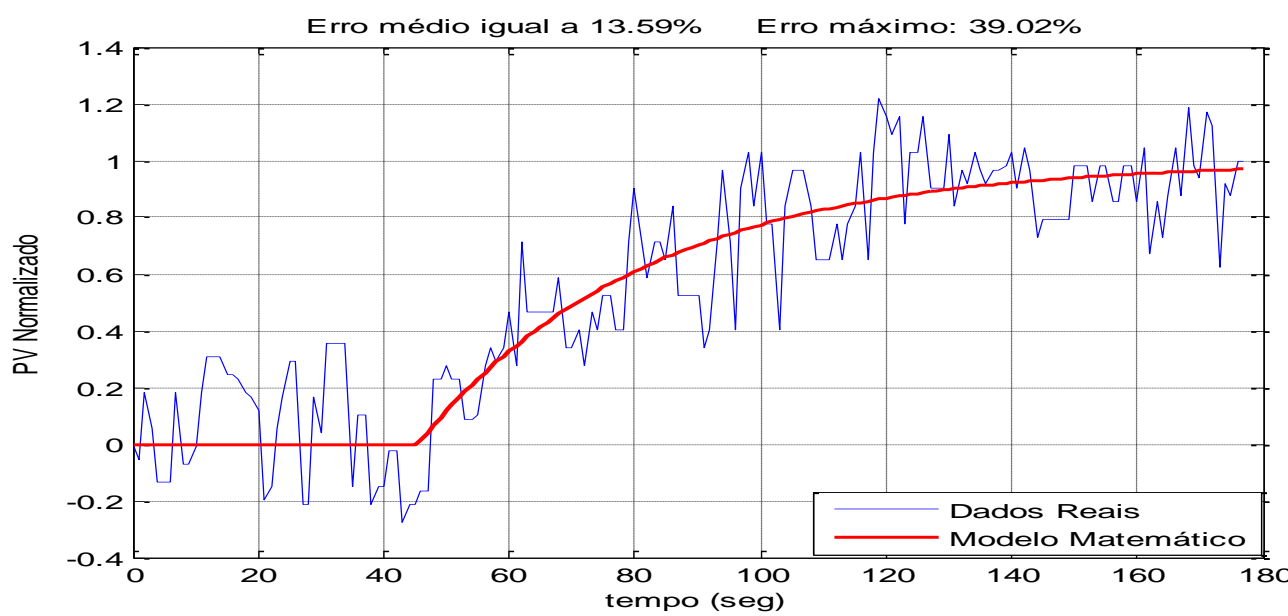

Figura 4 - Modelo real x matemático da parte II obtido para modelagem do controlador.

* Contribuição técnica ao $18^{\circ}$ Seminário de Automação e TI Industrial, 23 a 26 de setembro de 2014, São Paulo, SP, Brasil. 
De posse dos parâmetros do processo, foi utilizado o método da síntese direta para parametrização dos controladores PI.

Dadas as condições de operação do forno, onde o vapor tem que estar superaquecido, existe uma pressão máxima de trabalho para segurança e os acoplamentos existentes entre as malhas, será considerado para projeto um controlador conservador sem sobressinal. Mas, o conservadorismo tem que ser capaz de reduzir o tempo de acomodação do sistema em malha fechada e compensar a perturbação de característica integradora encontrada.

Baseado nessas premissas, o controlador ficou com os seguintes parâmetros:

Tabela 7: Parâmetros calculados para utilizar-se no controlador PI.

\begin{tabular}{ccc}
\hline Controlador PI & Kc & Ti \\
$\mathrm{C}(\mathrm{s}) 1^{2}$ Parte & $-0,29$ & $25,38 \mathrm{~s}$ \\
\hline
\end{tabular}

Depois de calculado os parâmetros PI do controlador, foi feito teste em malha fechada no controlador e como objetivos desejados para a malha são: redução do tempo de acomodação do sistema e eliminação do "offset" da PV sobre o SP na regulação do processo. Abaixo estão as respostas pré e pós re-sintonia do controlador com as respectivas análises:

a)

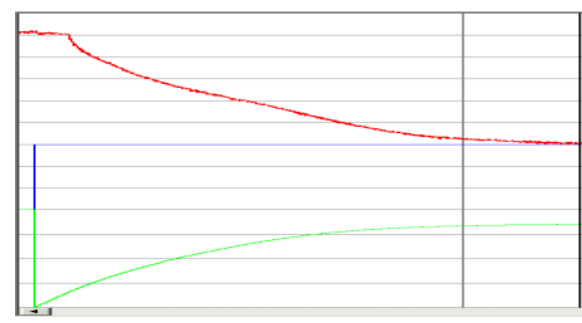

b)

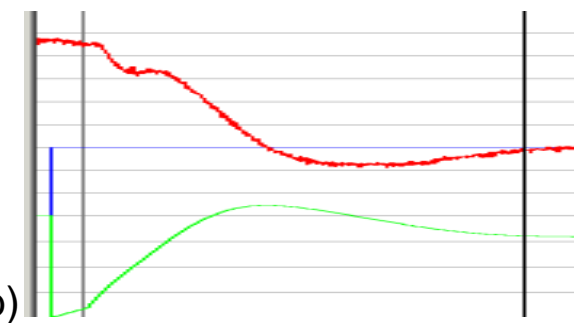

Figura 5 - Transitório no início do tratamento. a) pré re-sintonia; b) pós re-sintonia (pena vermelha $\mathrm{PV}$, pena verde - CO, pena azul - SP).

Com os parâmetros antigos do $\mathrm{PI}$, ganho $\mathrm{Kp}=-0,06$ e $\mathrm{Ti}=20 \mathrm{~s}$, o tempo de acomodação do sistema em malha fechada é de aproximadamente de cinquenta minutos à uma hora figura 25 - a. Com os parâmetros novos $\mathrm{Kp}=-0,29$ e $\mathrm{Ti}=25,38 \mathrm{~s} 0$ tempo de acomodação do sistema em malha fechada reduziu para aproximadamente vinte e cinco minutos figura 25 - b, porém, ocorreu um indesejável sobressinal. Esse sobressinal pode, porém, ser melhorado através da seguinte ação:

- Correção do tempo de execução do cálculo do bloco PID que pode estar traduzindo em um erro do ganho integral.

Após o transitório, na regulação notou-se a capacitação do controlador em compensar a perturbação existente no processo. As comparações seguem abaixo:

* Contribuição técnica ao $18^{\circ}$ Seminário de Automação e TI Industrial, 23 a 26 de setembro de 2014, São Paulo, SP, Brasil. 


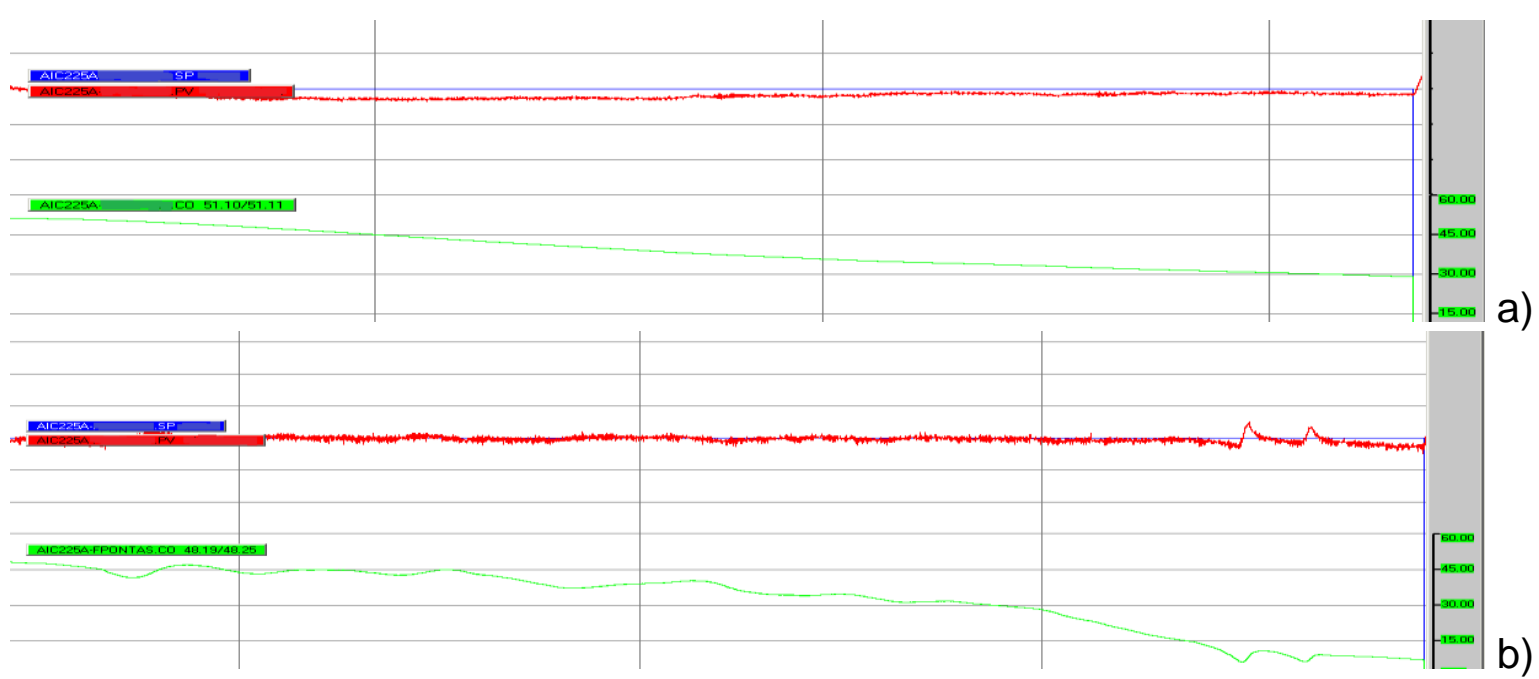

Figura 6 - Regulação do tratamento. a) pré re-sintonia; b) pós re-sintonia (pena vermelha - PV, pena verde - CO, pena azul - SP).

Com o controlador antigo, nota-se a existência de um erro de controle constante que compromete a regulação do processo figura 26- a. Já com o novo controlador, o erro de controle é eliminado, ou seja, a PV = SP.

Para consolidar e alcançar melhores retornos técnicos e consequentemente de qualidade do produto final mais efetivos, é necessário realizar as ações que estão propostas abaixo que abrangem estratégias de controle mais robustas, otimização das demais malhas e também a verificação de outros componentes do sistema como exemplo válvulas de controle.

Ações propostas

$1^{a}$ - Verificação da implementação do bloco de cálculo PID no algoritmo do CLP:

Realizar os ajustes necessários nos parâmetros de tempo de execução do cálculo e frequência da rotina de execução, os mantendo iguais.

$2^{\underline{a}}-$ Adoção da técnica de controle chaveado para a malha AIC 225

Como o processo é altamente não linear, já foi citado, estudado por alguns autores e obtido bons resultados a utilização do controlador chaveado [11].

$3^{\mathrm{a}}$ e $4^{\mathrm{a}}$ - Alteração da estratégia de controle para o controle de temperatura interna do forno no Patamar e utilização da compensação direta "feedfoward" para o TIC 410A, com o sinal do FCV 225A

Foi proposto a adoção de um controlador atuando diretamente nos dois aquecedores devido à necessidade de homogeneização da temperatura interna.

Para isso, a ideia proposta segue abaixo:

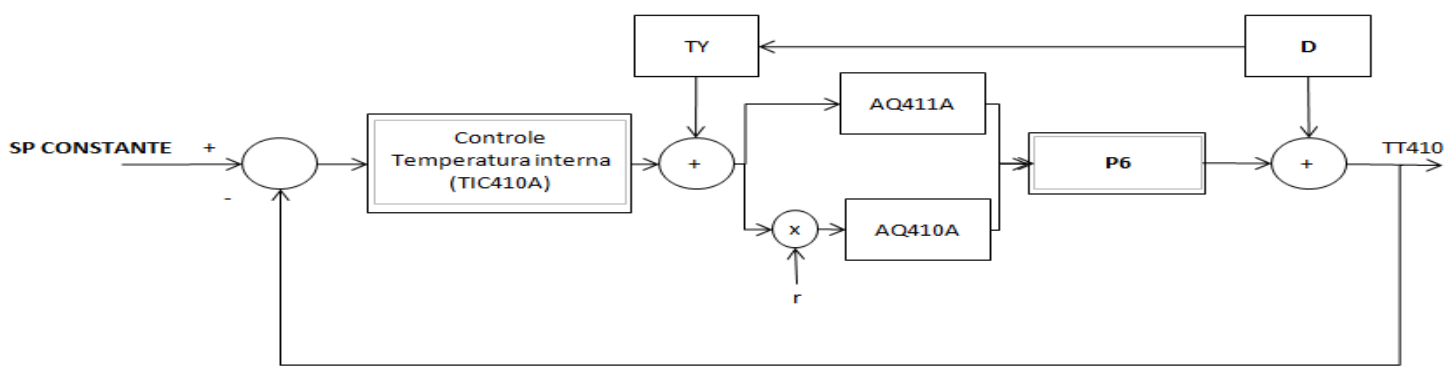

Figura 7 - Diagrama de blocos da estratégia proposta II para TIC 410A

A ideia desta estratégia é garantir a homogeneização da temperatura interna através do fator de multiplicação $r$ na potência necessária para compensação do fenômeno

* Contribuição técnica ao $18^{\circ}$ Seminário de Automação e TI Industrial, 23 a 26 de setembro de 2014, São Paulo, SP, Brasil. 
termodinâmico de convecção térmica e como o vapor que entra no forno é uma perturbação à temperatura interna, a ideia em utilizar a estratégia também conhecida como feedfoward em conjunto com a citada anteriormente, irá reduzir a propagação do erro gerado pela perturbação otimizando o controle.

5 - Verificação do atuador FCV 225A

Conforme levantado na etapa II, observou-se a presença de uma banda morta na válvula entre 0 à $10 \%$. Com isso, essa banda morta, reduz a rangeabilidade do atuador para de $10 \%$ à $100 \%$.

Como conclusão, sugere-se a análise do atuador pela equipe de manutenção/instrumentação para verificação da possibilidade de manutenção da válvula ou então a troca por um atuador linear, que pode ser justificada pela larga faixa de operação da válvula.

\subsection{Fase IV: Avaliação Final de Desempenho}

Variabilidade relativa da PV (varPV): $3,92 \%$

Integral do erro médio - IEM: $-0.017 \%$

Excursão da CO: 14.83 aberturas/dia

Índices para resposta ao degrau:

Tempo de acomodação: 27 minutos

Dos índices calculados, algumas análises podem ser feitas sobre o cenário pré e pós otimização:

VarPV antes $=2,49 \%$, pós $=3,92 \%$

Conforme figura $25-b$, possivelmente, o sobressinal existente após a sintonia do controlador e a região de não linearidade do atuador verificado no final do tratamento da figura 26 -b são responsáveis pelo aumento da distorção da PV em torno da média, com isso, o índice da variabilidade da PV foi afetado.

IEM - antes $=-0,066 \%$, pós $=-0,017 \%$

Com a nova sintonia do controlador AIC225A, conseguiu-se eliminar o "offset" existente na PV com o SP, visto que os novos parâmetros foram capazes de controlar o processo compensando a perturbação integradora existente, refletindo no índice de IEM.

Excursão da CO - antes $=2.89$ aberturas/dia, pós = 14.83 aberturas $/$ dia

Para eliminação do "offset" conforme foi feito, um controlador mais agressivo foi utilizado e como consequência, os efeitos da perturbação que antes eram percebidos na PV, foram compensados pelo controlador aumentando a excursão do atuador.

Tempo de acomodação: Ta antes $=51$ minutos, Ta pós $=27$ minutos

Um dos principais objetivos para a malha foi alcançado que era de reduzir o tempo de acomodação.

Para concluir, com os trabalhos realizados em um curto período melhoras técnicas foram notórias sendo que melhorias na qualidade ainda não foram possíveis mensurar devido ao curto período da realização do trabalho. Para potencializar essas melhorias, a realização das ações propostas devem ser feitas e podem ser citadas em um outro trabalho.

\section{CONCLUSÃo}

Pelo exposto na introdução, é alarmante a situação dos controladores regulatórios existentes hoje nas plantas industriais não apenas do Brasil mas em outros países.

* Contribuição técnica ao $18^{\circ}$ Seminário de Automação e TI Industrial, 23 a 26 de setembro de 2014, São Paulo, SP, Brasil. 
Após a realização deste projeto, fica evidente a possibilidade da reversão desse cenário para um mais satisfatório.

Para alcançar esses resultados, é necessária a adoção da cultura por parte das empresas visando capacitar profissionais para realização de trabalhos rotineiros de avaliação de desempenho dos sistemas e também de uma criteriosa auditoria das malhas conforme abordado.

Dadas as ações realizadas e propostas, é mostrado que um bom trabalho por parte de engenheiros de controle, adotando estratégias de controle, re-sintonia de controladores, revisões de implementações em CLP's já trariam ganhos técnicos e potenciais em qualidade do produto final. Porém, para que se atinja o "estado da arte", ações de manutenção corretiva, preventiva e preditiva em sensores e atuadores são necessárias visto que possuem vida útil limitada e sofrem degradações de desempenho ao longo do tempo.

Com todas essas informações já consolidadas e constatadas através deste e de outros trabalhos já realizados, aliados com a necessidade cada vez maior por parte das empresas em reduzir custos gerais de produção, espera-se tornar tendência o crescimento da demanda por profissionais qualificados para execução dessas atividades.

\section{Agradecimentos}

Agradeço à minha empresa por disponibilizarem tempo de meu trabalho para me dedicar a esse estudo de caso. Agradeço também à siderúrgica que permitiu e confiou à mim a capacidade de realizar a auditoria no forno de tratamento térmico.

\section{REFERÊNCIAS}

1 Brand FR. Modelo de inferência de variabilidade: traduzindo o desempenho de malhas de controle em alterações de variabilidade [Dissertação de mestrado]. Porto Alegre; 2009.

2 Fonseca $\mathrm{MO}$, et al. Avaliação de desempenho e auditoria de malhas de controle. InTech, ano VI, no 63, p. 32-37.

3 Passos LFD. Metodologia para Auditoria e validação ampla de malhas de controle Estudo de caso para uma caldeira industrial [Dissertação de mestrado].Belo Horizonte: Universidade Federal de Minas Gerais; 2009.

4 Farenzena M, et al. Gerenciamento de ativos: ferramentas para aumento da performance das unidades. Revista Controle e instrumentação, 115, pág. 89-95.

5 Seborg DE, et al. Process Dynamics and Control. John Wiley \& Sons. 1989.

6 Nunes TC. Práticas industriais para instrumentação e controle em circuitos de flotação mecânica [Monografia de pós-graduação]. Ouro Preto: Universidade Federal de Ouro Preto; 2010

7 Braga AR. Implementação de Estratégias de Controle Multimalha e Multivariável [Dissertação de mestrado].Belo Horizonte: Universidade Federal de Minas Gerais; 1994

8 Lira SA. Análise de correlação: abordagem teórica e de construção dos coeficientes com aplicações [Dissertação de mestrado]. Curitiba: Universidade Federal do Paraná; 2004.

9 Silva ESRL. Investigação do comportamento dinâmico e avaliação de estratégias de identificação, controle e otimização de um reator [Dissertação de mestrado]. Curitiba: Universidade Federal do Paraná;2006.

10 Baeta, ÉS. Implementação e análise comparativa de técnicas de controle PID em sistemas distribuídos e centralizados [Dissertação de mestrado]. Belo Horizonte: Universidade Federal de Minas Gerais; 2006.

\footnotetext{
* Contribuição técnica ao $18^{\circ}$ Seminário de Automação e TI Industrial, 23 a 26 de setembro de 2014, São Paulo, SP, Brasil.
} 
11 Pena PN. Aplicação de técnicas de controle chaveado a uma planta piloto: simulação e implementação[Dissertação de mestrado]. Belo Horizonte: Universidade Federal de Minas Gerais; 2002.

12 Guerra WA. Implementação de Controle Proporcional, Integral e Derivativo digital em controladores lógico programáveis [Monografia de pós graduação]. Recife: Universidade Federal de Pernambuco; 2009.

13 Costa PHM. Auditoria das malhas de controle de um forno de tratamento térmico siderúrgico [Monografia de graduação]. Belo Horizonte: Centro Universitário UNA; 2012.

* Contribuição técnica ao $18^{\circ}$ Seminário de Automação e TI Industrial, 23 a 26 de setembro de 2014, São Paulo, SP, Brasil. 